\title{
Perfil da força muscular isométrica em atletas de rugby em cadeira de rodas
}

\section{Profile of isometric muscle strength in athletes of wheelchair rugby}

(D)João Paulo Casteleti de Souza ${ }^{1}$, (D) Nayara Christine Souza ${ }^{1}$, (D)éssica Reis Buratti ${ }^{1}$, (DRicardo Aurélio Carvalho Sampaio ${ }^{1}$, (D)Ricardo Antônio Tanhoffer ${ }^{1}$, (D) Marco Carlos Uchida ${ }^{1}$, (D)José Irineu Gorla ${ }^{1}$

${ }^{1}$ Universidade Estadual de Campinas - UNICAMP

\section{Correspondência}

José Irineu Gorla

E-mail: gorla@fef.unicamp.br

Submetido: 15 Novembro 2019

Aceito: 02 Outubro 2020

\section{Como citar}

Souza JPC, Souza NC, Buratti JR, Sampaio RAC, Tanhoffer RA, Uchida MC, et al. Perfil da força muscular isométrica em atletas de rugby em cadeira de rodas. Acta Fisiatr. 2020;27(4):225232

DOI: 10.11606/issn.2317-0190.v27i4a168538

\section{RESUMO}

Objetivo: Analisar os níveis de força muscular em atletas de rugby em cadeira de rodas (RCR). Método: A amostra foi composta por 10 atletas (homens) com lesão da medula espinhal em nível cervical (tetraplegia) com média de idade de 31,1 \$5,06 anos. Os atletas foram recrutados na equipe de RCR do Projeto de Atividade Motora e Esporte Adaptado da Universidade Estadual de Campinas (ADEACAMP/UNICAMP). Resultados: Para analisar os níveis de força muscular isométrica (flexão e extensão dos ombros e cotovelos) foi utilizado dinamômetro isométrico, enquanto ultrassom foi usado para avaliação da espessura muscular (flexores e extensores do cotovelo, ambos os lados), utilizando 7,3 MHz da sonda linear-matriz. Correlação ponto-bisserial foi utilizada para verificar a associação entre os níveis de força de acordo com a posição de jogo; enquanto a correlação de Spearman verificou a associação entre os níveis de força entre membros dominantes e não dominantes, além da relação músculo agonista versus antagonista. Ainda, o teste Mann Whitney $U$ foi utilizado para comparar os jogadores titulares e reservas quanto à força e espessura muscular. Conclusão: Podemos concluir que quanto maior a classificação funcional, maiores são os valores de força voluntária isométrica máxima; e que a força isométrica tem correlação com o desempenho de atletas de RCR.

Palavras-chave: Esportes para Pessoas com Deficiência, Cadeiras de Rodas, Força Muscular, Dinamômetro de Força Muscular

\section{ABSTRACT}

Objective: The aim of this study was to analyze muscle strength levels in wheelchair rugby athletes. Methods: The sample was 10 athletes (men) with spinal cord injury at cervical level (tetraplegia) with mean age of $31.1 \pm 5.06$ years. The athletes were recruited in the rugby team in wheelchair of the Motor Activity and Adapted Sport Project of the University of Campinas (ADEACAMP/UNICAMP). Results: In order to analyze the isometric muscular strength levels (flexion/extension of the shoulders and elbows), dynamometer was used, while muscle thickness was verified by ultrasound (flexors and extensors of the elbow, both sides), using 7.3 MHz of the linear-array probe. Point biserial correlation was applied to verify associations of game position and strength; while Spearman's correlation verified associations of strength and dominant and nondominant sides, and the relationship of agonist versus antagonist muscles. Moreover, the Mann Whitney $U$ test was used to compare first to second-string players regarding strength and muscle thickness. Conclusion: We concluded that the higher the functional classification, higher the values of maximum isometric voluntary strength; and that the isometric strength correlates with their rugby performances.

Keywords: Sports for Persons with Disabilities, Wheelchairs, Muscle Strength, Muscle Strength Dynamometer 


\section{INTRODUÇÃO}

O Rugby em Cadeira de Rodas (RCR) surgiu no ano de 1977, na cidade de Winnipeg, Canadá, como uma alternativa de prática esportiva para pessoas com tetraplegia. ${ }^{1} \mathrm{O}$ RCR é jogado por duas equipes de quatro atletas titulares e no máximo oito reservas. Os atletas são divididos em sete classes, de acordo com sua mobilidade e resquícios de movimentos, quanto maior a motricidade, maior a pontuação; os atletas com classificações mais baixas jogam na defesa, e os com classificações mais altas, formam o ataque. Em quadra, a soma das classes dos jogadores não pode ultrapassar oito pontos em cada equipe. ${ }^{2}$

As dimensões da quadra tem $28 \mathrm{~m}$ de comprimento por 15 metros de largura e as pontuações (gols) são anotadas quando os atletas ultrapassam a linha de gol adversária com as duas rodas da cadeira e a bola nas mãos, ao mesmo tempo que devem evitar que a outra equipe obtenha a posse de bola e faça gols na linha de gol que está defendendo. ${ }^{2}$ Os praticantes de RCR são indivíduos com lesão da medula espinhal (LME) em nível cervical, advindos de doença congênita, degenerativa, trauma, infecção, isquemia, compressão por hematoma ou tumor, entre outros. Tal lesão compromete a transmissão dos impulsos nervosos, o que pode gerar alterações na sensibilidade e controle motor voluntário e involuntário; quanto mais cranial a lesão, mais graves são as sequelas. ${ }^{3,4}$

As principais alterações fisiológicas e neuromusculares da LME juntamente com a inatividade física está relacionada a perda da funcionalidade e das capacidades biomotoras de resistência, força e potência, a magnitude por sua vez está relacionada ao nível da lesão. ${ }^{5,6}$

Por esse motivo, o conjunto das alterações fisiológicas e neuromusculares da LME, juntamente com a inatividade física está relacionada à perda da funcionalidade e das capacidades biomotoras de resistência, força e potência. ${ }^{7,8}$ Assim, as fibras musculares tipo I, abaixo do nível da lesão se transformam em tipo Ilb, passam a ter características de maior trabalho anaeróbio, e esse processo se inicia entre 4-7 meses após a lesão. $^{9}$

Segundo Pelletier \& Hicks, ${ }^{8}$ a proporção do número de fibras musculares abaixo do nível da lesão é menor do que nos músculos com inervação preservada acima do nível da lesão. Assim sendo, possuem menor quantidade de proteínas contráteis e menores níveis máximos de força de contração. ${ }^{9}$

Devido as alterações morfológicas a musculatura abaixo do nível da lesão ainda pode sofrer de hipertonia, hipotonia ou atonia, dependendo do nível ou tipo de LME.,

Dessa forma, podemos dizer que acima do nível da lesão há a tendência da mudança do tipo de fibra muscular predominante para fibras do tipo I que tem características aeróbias, devido aos esforços relativos às atividades de vida diária e que abaixo do nível da lesão, a hipotrofia muscular ocorre em maior incidência, principalmente em casos de lesões completas. ${ }^{8}$ Considerando o exposto e a carência de pesquisas na literatura, o presente estudo teve como objetivo analisar os níveis de força muscular isométrica de atletas de RCR.

\section{OBJETIVO}

Considerando o exposto acima e a carência de pesquisas na literatura específica, este estudo teve como objetivo analisar os níveis de força muscular isométrica de atletas de RCR.

\section{MÉTODOS}

A amostra foi composta por 10 atletas com LME em nível cervical (tetraplegia), todos do sexo masculino com média de idade de $31,1 \pm 5,06$ anos. Os atletas pertencem à equipe de Rugby em Cadeira de Rodas do Projeto de Atividade Motora e Esporte Adaptado da Universidade Estadual de Campinas na ADEACAMP/UNICAMP, participaram da 1a Divisão do Campeonato Brasileiro desde a sua existência em 2008, conquistando um total de 4 títulos nacionais. Além disso, dos 10 atletas avaliados 4 fazem parte da seleção brasileira e disputaram os Jogos ParaPanamericanos de Toronto 2015.

Foram incluídos no estudo atletas com LME nível cervical há pelo menos um ano, e ser praticante de RCR há pelo menos um ano com regime semanal mínimo de 4 horas de treinamento, e não ter realizado nenhuma intervenção cirúrgica nos membros superiores. Foram excluídos da amostra aqueles que não se sentirem confortáveis durante a realização do estudo.

Todos os participantes assinaram o Termo de Consentimento Livre e Esclarecido para participação na pesquisa, que esteve em conformidade com os parâmetros de ética em pesquisa da Lei de ética em pesquisa envolvendo seres humanos no 466/2012, aprovado pelo Comitê de Ética de Pesquisa em estudos com seres humanos da Faculdade de Medicina da UNICAMP (Parecer CEP no 83875 - 27/08/2012) com adendo (Parecer CEP no 939.079 - 29/01/2015).

Tabela 1. Perfil demográfico dos atletas de RCR

\begin{tabular}{cccccccc}
\hline Atleta & CF & $\begin{array}{c}\text { Nível da } \\
\text { lesão }\end{array}$ & $\begin{array}{c}\text { Idade } \\
\text { (anos) }\end{array}$ & $\begin{array}{c}\text { Altura } \\
(\mathbf{m})\end{array}$ & $\begin{array}{c}\text { Peso } \\
\text { (Kg) }\end{array}$ & $\begin{array}{c}\text { Tempo } \\
\text { lesão } \\
\text { (anos) }\end{array}$ & $\begin{array}{c}\text { Tempo } \\
\text { treino } \\
\text { (anos) }\end{array}$ \\
\hline A & 0.5 & C5-C6 & 29 & 1,7 & 68,91 & 10 & 5 \\
B & 0.5 & C5-C6 & 27 & 1,93 & 78 & 7 & 6 \\
C & 1.0 & C6-C7 & 38 & 1,76 & 65,2 & 11 & 7 \\
D & 1.0 & C5-C6 & 25 & 1,8 & 90,35 & 5 & 2 \\
E & 2.0 & C6 & 30 & 1,8 & 78 & 13 & 2 \\
F & 2.0 & C6-C7 & 28 & 1,75 & 61,85 & 6 & 1,2 \\
G & 2.0 & C6-C7 & 36 & 1,7 & 61 & 10 & 6 \\
H & 2.5 & C7 & 28 & 1,75 & 71,05 & 11 & 7 \\
I & 2.5 & C6-C7 & 30 & 1,58 & 62 & 9 & 7 \\
J & 3.0 & C6-C7 & 40 & 1,75 & 76,2 & 6 & 2,6 \\
& Média & & 31,1 & 1,75 & 71,25 & 8,8 & 4,58 \\
& DP & & $\pm 5,06$ & $\pm 0,08$ & $\pm 9,43$ & 2,65 & $\pm 2,36$ \\
\hline
\end{tabular}

CF: classificação funcional; DP: desvio padrão

De acordo com Thomas et al. ${ }^{10}$, este estudo caracteriza-se como pesquisa descritiva e apresenta um delineamento transversal. Para o desenvolvimento deste estudo e com o intuito de analisar os níveis de força muscular isométrica, foi utilizado o Handheld Dynamometer (HHD; mTas F-1; ANIMA, Tokyo, Japão), o equipamento permite avaliar a força isométrica de qualquer movimento articular. Foi utilizado o HHD para avaliação da força isométrica nos movimentos de flexão e extensão do ombro e flexão e extensão do cotovelo. 0 HHD utiliza 3 unidades de medida de força: a Libra-força (lbf), o Quilo grama $(\mathrm{Kg})$ e o Newton (N). Nesse caso, optou por utilizar o Newton como unidade de medida, por ser a unidade de força apropriada. O HHD, além de avaliar o pico de torque (PT) também avalia o tempo (s) que o voluntário utiliza para chegar no PT. Houve preocupação por parte do avaliador em 
verificar se a calibração do equipamento estava correta.

O protocolo de referência utilizado foi o de Bohannon, ${ }^{11}$ onde criou valores de referência para força muscular isométrica em 10 movimentos articulares, sendo extensão de punho, flexão e extensão de cotovelo, rotação lateral, extensão e abdução de ombro, dorsiflexão plantar, extensão de joelho, abdução e flexão de quadril (Quadro 1).

Quadro 1. Protocolo de posicionamento proposto por Bohannon $(\mathrm{HHD})^{11}$

\begin{tabular}{|c|c|c|}
\hline $\begin{array}{l}\text { Movimento } \\
\text { Articular }\end{array}$ & $\begin{array}{l}\text { Posicionamento } \\
\text { articular }\end{array}$ & $\begin{array}{c}\text { Posicionamento } \\
\text { do HHD }\end{array}$ \\
\hline $\begin{array}{l}\text { Extensão de } \\
\text { punho }\end{array}$ & $\begin{array}{l}\text { ombro neutro, cotovelo } 90 \text {, } \\
\text { punho neutro }\end{array}$ & $\begin{array}{l}\text { próximo da articulação } \\
\text { metacarpofalangiana }\end{array}$ \\
\hline $\begin{array}{l}\text { Flexão de } \\
\text { cotovelo }\end{array}$ & $\begin{array}{l}\text { ombro neutro, cotovelo } 90 \% \text {, } \\
\text { punho supinado }\end{array}$ & $\begin{array}{l}\text { próximo do processo } \\
\text { estilóide }\end{array}$ \\
\hline $\begin{array}{l}\text { Extensão de } \\
\text { cotovelo }\end{array}$ & $\begin{array}{l}\text { ombro neutro, cotovelo } 90 \text {, } \\
\text { punho neutro }\end{array}$ & $\begin{array}{l}\text { próximo do processo } \\
\text { estilóide }\end{array}$ \\
\hline $\begin{array}{l}\text { Rotação lateral } \\
\text { de ombro }\end{array}$ & $\begin{array}{l}\text { ombro abdução } 45^{\circ} \text {, } \\
\text { cotovelo } 90 \text { 은 }\end{array}$ & $\begin{array}{l}\text { próximo do processo } \\
\text { estilóide }\end{array}$ \\
\hline $\begin{array}{l}\text { Extensão de } \\
\text { ombro }\end{array}$ & $\begin{array}{l}\text { Ombro flexão } 90 \text {, cotovelo } \\
\text { flexionado }\end{array}$ & $\begin{array}{l}\text { próximo do epicôndilo } \\
\text { umeral }\end{array}$ \\
\hline $\begin{array}{l}\text { Abdução de } \\
\text { ombro }\end{array}$ & $\begin{array}{l}\text { ombro abdução } 45 \text { ㅇ, } \\
\text { cotovelo extendido }\end{array}$ & $\begin{array}{l}\text { próximo do epicôndilo } \\
\text { umeral }\end{array}$ \\
\hline $\begin{array}{l}\text { Dorsiflexão } \\
\text { plantar }\end{array}$ & $\begin{array}{l}\text { quadril e joelho em } \\
\text { extensão, tornozelo neutro }\end{array}$ & $\begin{array}{l}\text { próximo da articulação } \\
\text { metatarsofalangiana }\end{array}$ \\
\hline $\begin{array}{l}\text { Extensão de } \\
\text { joelho }\end{array}$ & $\begin{array}{l}\text { quadril e joelho em flexão } \\
90 \text { 일 }\end{array}$ & próximo do malêolo \\
\hline $\begin{array}{l}\text { Abdução de } \\
\text { quadril }\end{array}$ & $\begin{array}{l}\text { ambos os quadrils neutro, } \\
\text { joelho extendido }\end{array}$ & $\begin{array}{l}\text { próximo do condilo } \\
\text { femural }\end{array}$ \\
\hline $\begin{array}{l}\text { Flexão de } \\
\text { quadril }\end{array}$ & $\begin{array}{l}\text { quadril flexão } 90 \text {, joelho } \\
\text { flexionado, quadril } \\
\text { contralateral neutro }\end{array}$ & $\begin{array}{l}\text { próximo da linha lateral } \\
\text { da articulação do joelho }\end{array}$ \\
\hline
\end{tabular}

Todos os voluntários foram avaliados em suas próprias cadeira de RCR. Para o movimento de extensão e flexão de ombro, o mesmo ficou em posição neutra com o cotovelo flexionado a aproximadamente 90․ A célula de carga ficou posicionada no ponto mais distal, próximo ao epicôndilo umeral. Para o movimento de flexão de cotovelo, o ombro ficou posicionado a aproximadamente 45으 de flexão, o cotovelo com flexão de 90 e e o antebraço na posição supina. A célula de carga ficou posicionada no ponto mais proximal do processo estilóide. O movimento de extensão do cotovelo utilizou o mesmo posicionamento do movimento de flexão do cotovelo, apenas com alteração do antebraço que ficou na posição neutra. O Quadro 2 refere-se aos posicionamentos utilizados neste estudo.

Quadro 2. Protocolo de posicionamento proposto para RCR (HHD)

\begin{tabular}{|c|c|c|}
\hline $\begin{array}{l}\text { Movimento } \\
\text { Articular }\end{array}$ & $\begin{array}{l}\text { Posicionamento } \\
\text { articular }\end{array}$ & $\begin{array}{c}\text { Posicionamento } \\
\text { do HHD }\end{array}$ \\
\hline $\begin{array}{l}\text { Flexão de } \\
\text { cotovelo }\end{array}$ & $\begin{array}{l}\text { ombro aproximadamente } \\
45 \circ \text {, cotovelo } 90 \circ \text { apoiado } \\
\text { no joelho, punho neutro }\end{array}$ & $\begin{array}{l}\text { próximo do processo } \\
\text { estiloide }\end{array}$ \\
\hline $\begin{array}{l}\text { Extensão de } \\
\text { cotovelo }\end{array}$ & $\begin{array}{l}\text { ombro aproximadamente } \\
45^{\circ} \text {, cotovelo } 90 \% \text { apoiado } \\
\text { no joelho, punho supinado }\end{array}$ & $\begin{array}{l}\text { próximo do processo } \\
\text { estiloide }\end{array}$ \\
\hline $\begin{array}{l}\text { Flexão de } \\
\text { ombro }\end{array}$ & $\begin{array}{l}\text { ombro neutro, cotovelo } \\
\text { aproximadamente } 900\end{array}$ & $\begin{array}{l}\text { porção distal anterior do } \\
\text { úmero, }\end{array}$ \\
\hline $\begin{array}{l}\text { Extensão de } \\
\text { ombro }\end{array}$ & $\begin{array}{l}\text { ombro neutro, cotovelo } \\
\text { aproximadamente } 900\end{array}$ & $\begin{array}{l}\text { próximo do epicôndilo } \\
\text { umeral }\end{array}$ \\
\hline
\end{tabular}

O protocolo utilizado foi de 3 séries de contração isométrica voluntária máxima com 1 repetição em cada.
O intervalo entre as repetições foi de 120 segundos. 0 mesmo protocolo foi utilizado nos 4 movimentos articulares (flexão de cotovelo, extensão de cotovelo, flexão de ombro e extensão de ombro). Para análise estatística utilizou-se o maior valor dentre as tentativas (Quadro 3).

Quadro 3. Protocolo do teste de força isométrica (HHD)

\begin{tabular}{|llccc|}
\hline $\begin{array}{c}\text { Movimento } \\
\text { Articular }\end{array}$ & $\begin{array}{c}\text { Tipo de } \\
\text { contração }\end{array}$ & $\begin{array}{c}\text { No } \\
\text { series }\end{array}$ & $\begin{array}{c}\text { No } \\
\text { repetições }\end{array}$ & $\begin{array}{c}\text { Intervalo } \\
\text { entre séries }\end{array}$ \\
\hline Flexão do cotovelo & Isométrica & 3 & 1 & $120 \mathrm{~s}$ \\
Extensão do cotovelo & Isométrica & 3 & 1 & $120 \mathrm{~s}$ \\
Flexão do cotovelo & Isométrica & 3 & 1 & $120 \mathrm{~s}$ \\
Extensão do cotovelo & Isométrica & 3 & 1 & $120 \mathrm{~s}$ \\
\hline
\end{tabular}

Para avaliação da espessura muscular foi utilizado o Ultrassom da marca SonoSite ${ }^{\circledR}$ (HDI-3000, ATL, Bothell, Washington, EUA), utilizando 7,3 $\mathrm{MHz}$ da sonda linear-matriz.

As mesurações dos músculos flexores e extensores do cotovelo foram realizadas no membro dominante e não dominante. A mensuração da espessura dos músculos flexores do cotovelo (biceps e braquial) foram realizadas após os sujeitos ficarem 20 minutos na posição supina para que ocorra a troca de fluidos. ${ }^{12}$

O protocolo utilizado foi o de Abe et al. ${ }^{13}$, em que o ponto de posicionamento do transdutor para coleta da imagem se localiza a $60 \%$ distal entre o acromio e o epicondilo lateral do umero. Para que não houvesse diferença de mensuração do músculo, todos os sujeitos tiveram um posicionamento de braço a 45ㅇ de abdução de ombro (goniometria manual).

Para mesuração do músculo extensor do cotovelo, os atletas ficaram sentados em suas próprias cadeiras de rodas de uso diário e o braço se manteve paralelo ao tronco, o posicionamento do transdutor para coleta da imagem também foi posicionado a $60 \%$ distal entre o acrômio e o epicôndilo lateral do úmero. ${ }^{14}$

Durante todas as medições os sujeitos foram intruídos a relaxar os músculos dos braços, o transdutor foi alinhado perpendicularmente ao músculo avaliado. $O$ avaliador teve $o$ cuidado de durante a varredora manter uma pressão mínima evitando a compressão do músculo.

Os dados foram analisados através de estatística descritiva e inferencial. Com a estatística descritiva foram apresentados os valores dos níveis de força muscular de flexão e extensão de ombros; flexão e extensão de cotovelos; e espessura dos músculos flexores do cotovelo estão apresentados em gráficos e tabelas. Para todas as variáveis, os valores estão expressos em média \pm desvio padrão.

Utilizou-se o teste correlacional point-biserial para verificar a associação entre os níveis de força de atletas de RCR de acordo com a posição de jogo (defesa e ataque), e o teste de correlação de Spearman (Spearman's rho) para verificar a associação entre os níveis de força nos membros dominantes e não dominantes; e relação agonista versus antagonista.

O teste Mann Whitney $U$ foi utilizado para comparar os jogadores titulares e reservas quanto à força dos músculos flexores e extensores dos ombros; flexores extensores dos cotovelos; e espessura dos músculos flexores e extensores do cotovelo. A significância estatística foi determinada como $\mathrm{p} \leq 0,05$ e para todas as análises foi utilizado o Statistical Package for the Social Sciences (versão 21.0, SPSS, IBM Inc., Chicago, IL, USA). 


\section{RESULTADOS}

As Tabelas 2 e 3 mostram os resultados da avaliação de força muscular isométrica para os movimentos de flexão e extensão do ombro e flexão e extensão do cotovelo com o HHD, onde a média do tempo realizado para execução dos movimentos foi $3.06 \pm 1.03 \mathrm{~s}$. Na Tabela 4 está apresentada a média da espessura dos músculos flexores e extensores do cotovelo através do US.

Tabela 2. Força muscular nos movimentos de flexão e extensão do ombro

\begin{tabular}{|c|c|c|c|c|c|}
\hline Atleta & CF & $\begin{array}{c}\text { FOD } \\
(\mathrm{N})\end{array}$ & $\begin{array}{c}\text { FOE } \\
(N)\end{array}$ & $\begin{array}{c}\text { EOD } \\
(\mathrm{N})\end{array}$ & $\begin{array}{c}\text { EOE } \\
(N)\end{array}$ \\
\hline$A$ & 0.5 & 86 & 56 & 36 & 43 \\
\hline $\mathrm{B}^{*}$ & 0.5 & 270 & 229 & 128 & 149 \\
\hline$C^{*}$ & 1.0 & 363 & 403 & 143 & 140 \\
\hline $\mathrm{D}$ & 1.0 & 270 & 243 & 89 & 107 \\
\hline$E$ & 2.0 & 375 & 446 & 174 & 199 \\
\hline $\mathrm{F}$ & 2.0 & 297 & 311 & 102 & 100 \\
\hline G & 2.0 & 318 & 326 & 139 & 182 \\
\hline $\mathrm{H}^{*}$ & 2.5 & 552 & 405 & 156 & 186 \\
\hline$I^{*}$ & 2.5 & 415 & 496 & 237 & 247 \\
\hline$J$ & 3.0 & 282 & 281 & 94 & 40 \\
\hline \multicolumn{2}{|c|}{ Média } & 322.80 & 319.60 & 129.80 & 139.30 \\
\hline \multicolumn{2}{|c|}{ DP } & \pm 119.89 & \pm 127.58 & \pm 54.68 & \pm 67.48 \\
\hline
\end{tabular}

CF: Classificação Funcional; FOD: Flexão de Ombro Direito; FOE: Flexão de Ombro Esquerdo; EOD: Extensão de Ombro Direito; EOE: Extensão de Ombro Esquerdo

Tabela 3. Força muscular isométrica nos movimentos de flexão e extensão do cotovelo

\begin{tabular}{|c|c|c|c|c|c|}
\hline Atleta & CF & $\begin{array}{l}\text { FCD } \\
\text { (N) }\end{array}$ & $\begin{array}{l}\text { FCE } \\
\text { (N) }\end{array}$ & $\begin{array}{l}\text { ECD } \\
\text { (N) }\end{array}$ & $\begin{array}{l}\text { ECE } \\
\text { (N) }\end{array}$ \\
\hline$A$ & 0.5 & 122 & 117 & 0 & 0 \\
\hline $\mathrm{B}^{*}$ & 0.5 & 142 & 161 & 0 & 0 \\
\hline$C^{*}$ & 1.0 & 208 & 216 & 29 & 48 \\
\hline D & 1.0 & 205 & 196 & 0 & 0 \\
\hline$E$ & 2.0 & 228 & 232 & 113 & 110 \\
\hline $\mathrm{F}$ & 2.0 & 210 & 187 & 31 & 66 \\
\hline G & 2.0 & 169 & 171 & 63 & 39 \\
\hline $\mathrm{H}^{*}$ & 2.5 & 175 & 228 & 140 & 140 \\
\hline$I^{*}$ & 2.5 & 189 & 194 & 202 & 201 \\
\hline $\mathrm{J}$ & 3.0 & 244 & 221 & 145 & 90 \\
\hline \multicolumn{2}{|c|}{ Média } & 189.20 & 192.30 & 72.30 & 69.40 \\
\hline \multicolumn{2}{|c|}{ DP } & \pm 37.85 & \pm 35.56 & \pm 72.79 & \pm 66.84 \\
\hline
\end{tabular}

CF-Classificação Funcional; FCD: Flexão de Cotovelo Direito; FCE: Flexão de Cotovelo Esquerdo; ECD: Extensão de Cotovelo Direito; ECE: Extensão de Cotovelo Esquerdo

Tabela 4. Espessura muscular dos músculos flexores e extensores do cotovelo

\begin{tabular}{|c|c|c|c|c|c|}
\hline Atleta & CF & $\begin{array}{l}\text { FCD } \\
(\mathrm{cm})\end{array}$ & $\begin{array}{c}\text { FCE } \\
(\mathrm{cm})\end{array}$ & $\begin{array}{l}\text { ECD } \\
(\mathrm{cm})\end{array}$ & $\begin{array}{c}\text { ECE } \\
(\mathrm{cm})\end{array}$ \\
\hline$A$ & 0.5 & 3.32 & 3.44 & 1.33 & 1.31 \\
\hline $\mathrm{B}^{*}$ & 0.5 & 3.74 & 4.14 & 1.27 & 1.21 \\
\hline$C^{*}$ & 1.0 & 3.68 & 3.49 & 1.97 & 2.32 \\
\hline $\mathrm{D}$ & 1.0 & 3.82 & 4.01 & 3.02 & 2.70 \\
\hline$E$ & 2.0 & 3.99 & 3.66 & 1.97 & 2.01 \\
\hline$F$ & 2.0 & 3.38 & 3.80 & 2.01 & 2.79 \\
\hline G & 2.0 & 3.53 & 3.78 & 1.56 & 1.40 \\
\hline $\mathrm{H}^{*}$ & 2.5 & 3.50 & 3.70 & 2.54 & 2.37 \\
\hline$I^{*}$ & 2.5 & 3.32 & 3.28 & 2.73 & 2.54 \\
\hline $\mathrm{J}$ & 3.0 & 4.52 & 4.54 & 2.01 & 1.96 \\
\hline \multicolumn{2}{|c|}{ Média } & 3.68 & 3.78 & 2.04 & 2.06 \\
\hline \multicolumn{2}{|c|}{ DP } & \pm 0.36 & \pm 0.36 & \pm 0.57 & \pm 0.58 \\
\hline
\end{tabular}

CF: Classificação Funcional; FCD: Flexão de Cotovelo Direito; FCE: Flexão de Cotovelo Esquerdo; ECD: Extensão de Cotovelo Direito; ECE: Extensão de Cotovelo Esquerdo

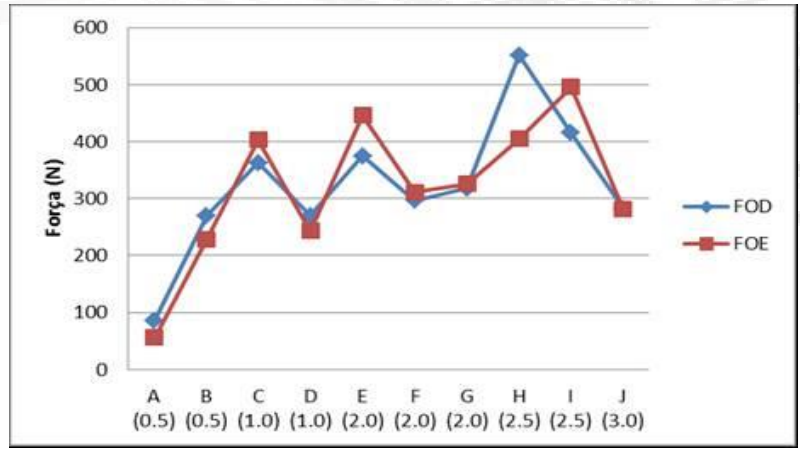

FOD: Flexão de Ombro Direito; FOE: Flexão de Ombro Esquerdo

Figura 1. Força muscular isométrica no movimento de flexão do ombro de acordo com a classificação funcional

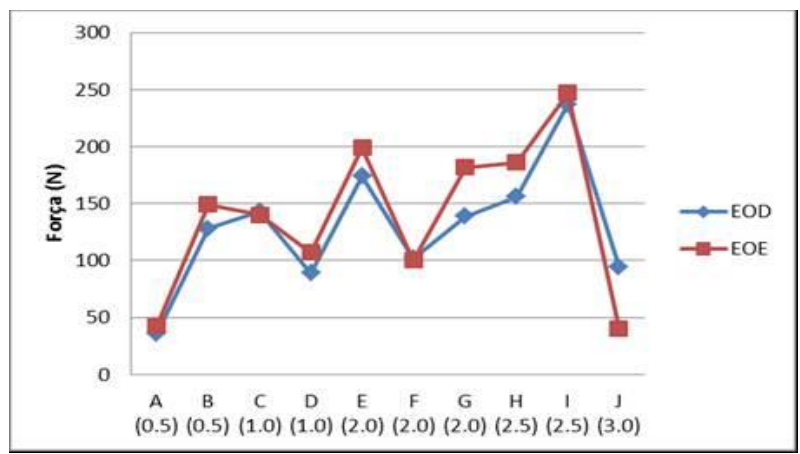

EOD - Extensão de Ombro Direito; EOE: Extensão de Ombro Esquerdo

Figura 2. Força muscular isométrica no movimento de extensão do ombro de acordo com a classificação funcional

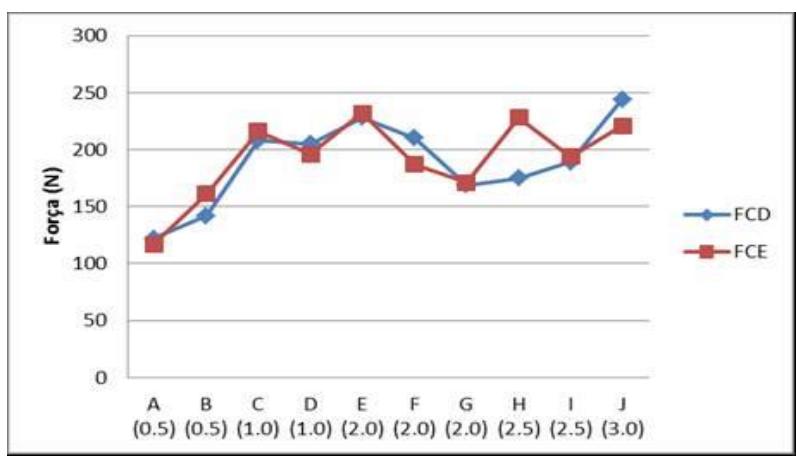

FCD: Flexão de Cotovelo Direito; FCE: Flexão de Cotovelo Esquerdo

Figura 3. Força muscular isométrica no movimento de flexão do cotovelo de acordo com a classificação funcional

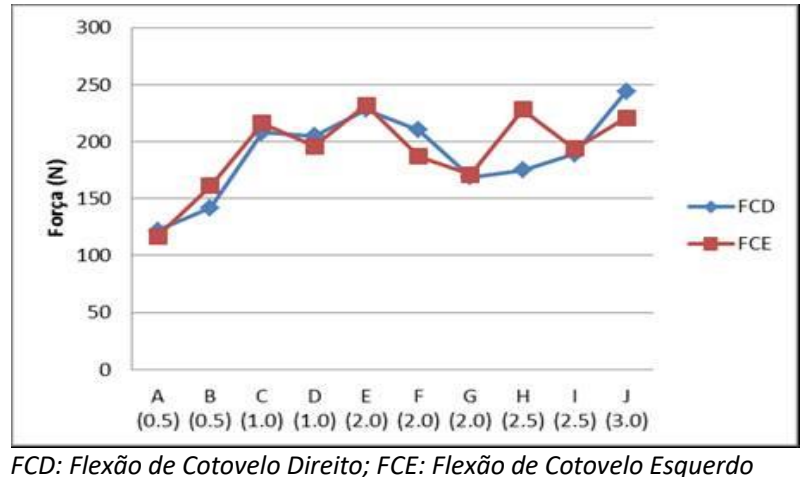

Figura 4. Força muscular isométrica no movimento de extensão do cotovelo de acordo com a classificação funcional 


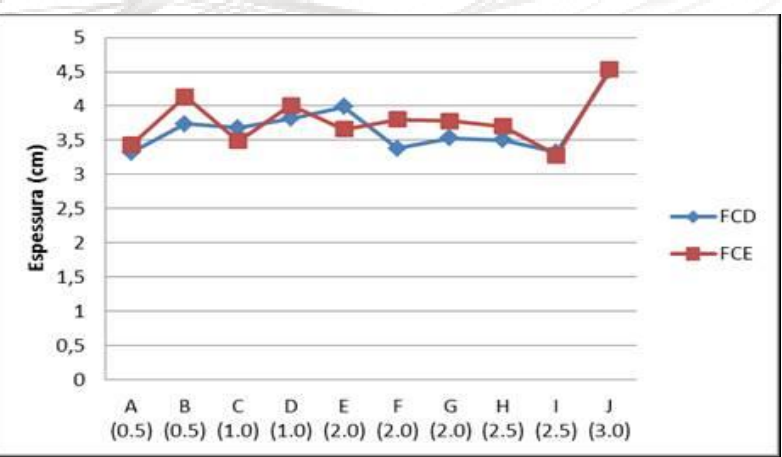

FCD: Flexão de Cotovelo Direito; FCE: Flexão de Cotovelo Esquerdo

Figura 5. Espessura muscular dos músculos flexores do cotovelo de acordo com a classificação funcional

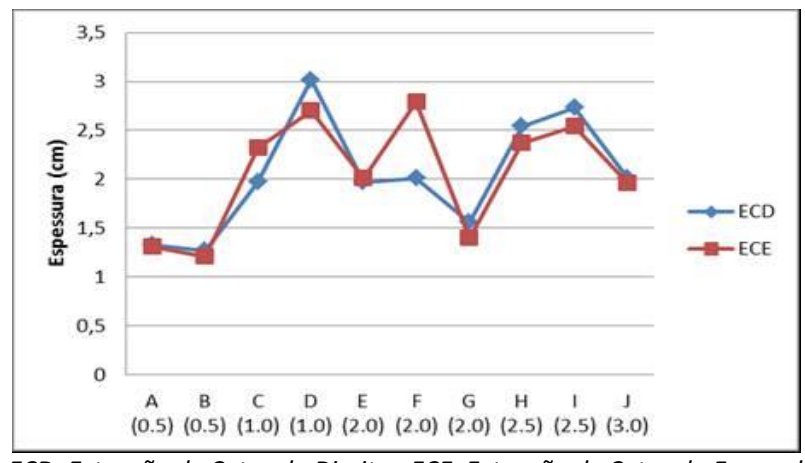

ECD: Extensão de Cotovelo Direito; ECE: Extensão de Cotovelo Esquerdo

Figura 6. Espessura muscular dos músculos extensores do cotovelo de acordo com a classificação funcional

A Tabela 5 apresenta uma correlação bi-serial (point biserial coefficient) entre os atletas que jogam na posição de defesa (Classes 0.5, 1.0 e 1.5) e os atletas que jogam na posição de ataque (Classificação Funcional 2.0, 2.5, 3.0).

Tabela 5. Correlação bi-serial entre as posições de defesa e ataque e níveis de força muscular

\begin{tabular}{ccc}
\hline Variáveis & Coeficiente de correlação $(\mathbf{r})$ & $\mathbf{P}$ \\
\hline FOD & 0.54 & 0.10 \\
FOE & 0.58 & 0.07 \\
EOD & 0.48 & 0.15 \\
EOE & 0.37 & 0.28 \\
FCD & 0.45 & 0.18 \\
FCE & 0.47 & 0.16 \\
ECD & 0.76 & $0.009^{* *}$ \\
ECE & 0.73 & $0.01 *$ \\
\hline
\end{tabular}

${ }^{*} p<0.05,{ }^{* *} p<0.01$; FOD: Flexão de Ombro Direito; FOE: Flexão de Ombro Esquerdo; EOD: Extensão de Ombro Direito; EOE: Extensão de Ombro Esquerdo; FCD: Flexão de Cotovelo Direito; FCE: Flexão de Cotovelo Esquerdo; ECD: Extensão de Cotovelo Direito; ECE: Extensão de Cotovelo Esquerdo

A Tabela 6 apresenta a correlação entre os níveis de força muscular isométrica nos membros dominantes e não dominantes pelo teste de correlação de Spearman para variáveis não paramétricas. A Tabela 7 apresenta a correlação entre os níveis de força muscular isométrica dos músculos agonistas e antagonistas pelo teste de correlação de Spearman para variáveis não paramétricas.
Tabela 6. Correlação de Spearman para níveis de força muscular entre membro dominante e não dominante

\begin{tabular}{ccc}
\hline Variáveis & Coeficiente de correlação $(\mathbf{r})$ & $\mathbf{P}$ \\
\hline FOD X FOND & 0.96 & $<0.001^{* * *}$ \\
EOD X EOND & 0.90 & $<0.001^{* * *}$ \\
FCD X FCND & 0.72 & $0.01^{*}$ \\
ECD X ECND & 0.92 & $<0.001^{*}$ \\
\hline
\end{tabular}

${ }^{*} p<0.05,{ }^{* *} p<0.001$; FOD: Flexão de Ombro Dominante; FOND: Flexão de Ombro Não Dominante; EOD: Extensão de Ombro Dominante, EOND: Extensão de Ombro Não Dominante, FCD: Flexão de Cotovelo Dominante; FCND: Flexão de Cotovelo Não Dominante; ECD: Extensão de Cotovelo Dominante; ECND: Extensão de Cotovelo Não Dominante

Tabela 7. Correlação de Spearman para níveis de força muscular isométrica entre músculos agonistas e antagonista

\begin{tabular}{ccc}
\hline Variáveis & Coeficiente de correlação $(\mathbf{r})$ & $\mathbf{P}$ \\
\hline FOD X EOD & 0.91 & $<0.001^{* * *}$ \\
FOE X EOE & 0.78 & $0.008^{* *}$ \\
FCD X ECD & 0.44 & 0.19 \\
FCE X ECE & 0.65 & $0.03^{*}$ \\
\hline
\end{tabular}

${ }^{*} p<0.05,{ }^{* *} p<0.01,{ }^{* * *} p<0.001 ;$ FOD: Flexão de Ombro Direito; FOE: Flexão de Ombro Esquerdo; EOD: Extensão de Ombro Direito; EOE: Extensão de Ombro Esquerdo; FCD: Flexão de Cotovelo Direito; FCE: Flexão de Cotovelo Esquerdo; ECD: Extensão de Cotovelo Direito; ECE: Extensão de Cotovelo Esquerdo

A Tabela 8 apresenta a comparação bivariada entre os jogadores titulares e os reservas, tanto em força muscular isométrica dos flexores e extensores do ombro e flexores e extensores do cotovelo quanto em espessura dos músculos flexores e extensores do cotovelo, todos os resultados apresentados em média e desvio padrão. Os jogadores titulares são: C (1.0), E (2.0), H (2.5) e I (2.5), formando o total de 8 pontos em quadra permitido pelo regulamento da IWRF.

Tabela 8. Comparação bivariada (Teste Mann Whitney U) entre jogadores de titulares e reservas

\begin{tabular}{lccc}
\hline \multicolumn{1}{c}{ Variáveis } & \multicolumn{2}{c}{ Média \pm DP } & \multirow{2}{*}{ Titulares $(\mathbf{n}=4)$} \\
& $426,25 \pm 86,73 \mathrm{~N}$ & $253,83 \pm 84,21 \mathrm{~N}$ & $0.01^{*}$ \\
\hline FOD HHD & $437,50 \pm 43,74 \mathrm{~N}$ & $241 \pm 98,07 \mathrm{~N}$ & $0.01^{*}$ \\
FOE HHD & $177,50 \pm 41,63 \mathrm{~N}$ & $98 \pm 36,16 \mathrm{~N}$ & $0.01^{*}$ \\
EOD HHD & $193 \pm 44 \mathrm{~N}$ & $103,50 \pm 56,47 \mathrm{~N}$ & $0.03^{*}$ \\
EOE HHD & $200 \pm 23,05 \mathrm{~N}$ & $182 \pm 45,88 \mathrm{~N}$ & 0.52 \\
FCD HHD & $217,50 \pm 17,07 \mathrm{~N}$ & $175,50 \pm 35,42 \mathrm{~N}$ & $0.05^{*}$ \\
FCE HHD & $121 \pm 71,76 \mathrm{~N}$ & $39,83 \pm 57,33 \mathrm{~N}$ & 0.13 \\
ECD HHD & $124,75 \pm 63,65 \mathrm{~N}$ & $32,50 \pm 39,08 \mathrm{~N}$ & $0.03^{*}$ \\
ECE HHD & $3,62 \pm 0,28 \mathrm{~cm}$ & $3,71 \pm 0,43 \mathrm{~cm}$ & 0.74 \\
FCD Ultrassom & $3,53 \pm 0,19 \mathrm{~cm}$ & $3,95 \pm 0,37 \mathrm{~cm}$ & $0.05^{*}$ \\
FCE Ultrassom & $2,30 \pm 0,39 \mathrm{~cm}$ & $1,86 \pm 0,64 \mathrm{~cm}$ & 0.39 \\
ECD Ultrassom & $2,31 \pm 0,22 \mathrm{~cm}$ & $1,89 \pm 0,70 \mathrm{~cm}$ & 0.39 \\
\hline ECE Ultrassom & &
\end{tabular}

${ }^{*} p<0.05$. HHD - Hand-Held Dynamometry; FOD: Flexão de Ombro Direito; FOE: Flexão de Ombro Esquerdo; EOD: Extensão de Ombro Direito, EOE: Extensão de Ombro Esquerdo, FCD: Flexão de Cotovelo Direito; FCE: Flexão de Cotovelo Esquerdo; ECD: Extensão de Cotovelo Direito; ECE: Extensão de Cotovelo Esquerdo 


\section{DISCUSSÃO}

Diversos autores tem estudado a modalidade do RCR em diversas áreas. Campana ${ }^{15}$ realizou um estudo com o intuito de estruturar o RCR nas suas funções técnicas e táticas, Campos et al. ${ }^{16}$ apresentou um ensaio fornecendo indicativos para o treinamento do RCR a partir de alterações fisiológicas, neuromusculares e bioquímicas de atletas de RCR.

Segundo Gouveia ${ }^{17}$ a potência pico e potência média estão próximas dos seus pares de classificação funcional e os atletas de defesa brasileiros apresentaram valores próximos às médias dos atletas poloneses, ${ }^{18}$ apenas no percentual de fadiga que os atletas de ataque brasileiros não conseguiram alcançar os valores máximos. Além de Gouveia ${ }^{17}$ e Morgulec-Adamowicz et. al. ${ }^{18}$, outros autores ${ }^{19,20}$ estudam o desempenho do metabolismo anaeróbio no RCR.

Yilla \& Sheerrill ${ }^{21}$ desenvolveram um teste de habilidades motoras (Bateria Beck) para praticantes de RCR que foi validada no Brasil por Gorla et al. ${ }^{22}$ As habilidades testadas são: teste de manejo de bola, precisão de passe, desempenho de bloqueio, velocidade em 20 metros e passe de longa distância.

A capacidade aeróbia foi avaliada por Poulain et al. ${ }^{23}$ que confirmou a reprodutibilidade no teste de esforço máximo Leger, Boucher para pessoas com lesão da medula espinhal. Já para Hartung et al. ${ }^{24}$ o teste de esteira, com o protocolo de acréscimo de velocidade e inclinação, foi considerado o melhor teste para avaliação aeróbia em pessoas com lesão da medula espinhal.

Dessa forma, percebemos que o RCR foi estudado em diversas áreas, porém não foi encontrado nenhum estudo no que diz respeito à força muscular em membros superiores utilizando a mesma metodologia descrita neste estudo. Para cumprir com o objetivo desse estudo "analisar os níveis de força muscular de atletas de RCR", e colaborar com o aumento de informações a respeito da modalidade, o equipamento HHD teve grande valia, onde conseguimos mensurar a força isométrica máxima nos movimentos de flexão e extensão de ombro e flexão e extensão de cotovelo com os atletas em condição próxima da prática esportiva, ou seja, com os atletas em suas próprias cadeiras de RCR e com todos os equipamentos utilizados na mesma. Além disso, conseguimos com o HHD um resultado tão confiável quanto com outro dinamômetro. ${ }^{25}$

Corroborando com o estudo foi feita a mensuração da espessura dos músculos flexores e extensores do cotovelo com - US portátil que também facilita a avaliação por sua praticidade, além de sua confiabilidade de resultados. ${ }^{26}$

O principal teste da parte clínica da classificação funcional é o teste manual de força muscular "Manual Muscle Test" MMT. ${ }^{27}$ Dessa forma, há uma crescente nos resultados dos grupos musculares preservados desde a classe 0.5 até a classe 3.0 como já foi apresentado na revisão bibliográfica desse estudo. Assim sendo, quando analisamos o melhor atleta em cada classe também encontramos uma crescente nos resultados do teste de força muscular isométrica máxima em todos os movimentos de ombro (FOD, FOE, EOD e EOE) exceto entre o atleta J (3.0) e os atletas $\mathrm{H} \mathrm{e} \mathrm{I} \mathrm{(ambos} \mathrm{2.5),} \mathrm{onde} \mathrm{o} \mathrm{atleta}$ J apresenta menores valores nos movimentos de FOD, FOE, $E O D, E O E, E C D$ e ECE, que pode ser explicado devido ao tempo de treinamento e desempenho esportivo individual.
Os atletas $\mathrm{H}$ e $\mathrm{J}$ treinam há mais tempo que o atleta J, além de serem atletas titulares da equipe ADEACAMP e atletas da seleção brasileira.

Já quando analisamos o atleta G (2.0) em comparação com o atleta $C$ (1.0) percebemos que o atleta $G$ executa menos força muscular isométrica máxima nos movimentos de FOD, FOE, $E O D, E O E$ e FCE que o atleta $C$ e tendo um resultado aproximado nos movimentos de FCD, ECD e ECE, mesmo tendo um tempo de treino aproximado. Dessa forma, o resultado encontrado pode indicar que $\mathrm{O}$ atleta $\mathrm{C}$ tem melhor desempenho esportivo que do atleta $G$, mas traz suspeita de que $o$ atleta $G$ pode ter uma funcionalidade próxima ou menor do que a do atleta C. Essa informação é de extrema importância para o atleta e sua comissão técnica, podendo usar os resultados desse teste para realizar um pedido de revisão de sua classificação funcional, confirmando se sua funcionalidade condiz com a funcionalidade de sua classe atual.

$O$ atleta $A(0.5)$ apresenta os menores resultados em todos os movimentos (FOD, FOE, EOD, EOE, FCD, FCE, ECD e ECE). Mesmo o atleta $B(0.5)$ sendo da mesma classe que o atleta $A$, sua força isométrica máxima foi 3 vezes maior para 0 movimento de FOD, 4 vezes maior para o movimento de FOE e aproximadamente 4 vezes maior para os movimentos de EOD e EOE. Esses resultados podem ser explicados pelo melhor desempenho esportivo do atleta $B$, porém pode ser levado em consideração que cada classe funcional tem uma "largura" (ex: o atleta da classe 0.5 pode apresentar de 0 a 5 no MMT para os músculos deltóide, peitoral superior, bíceps e extensores de punho. Dessa forma, o atleta B pode estar na "borda superior" (vantagem funcional dentro da classe) e o atleta A na "borda inferior" (desvantagem funcional dentro da classe).

Ao analisar os movimentos de FCD e FCE os valores são crescentes entre todas as classes e não discrepantes entre nenhum atleta. Esses resultados podem ser explicados uma vez que a LME de nenhum atleta foi alta suficiente para comprometer os músculos responsáveis pela flexão do cotovelo (braquial e bíceps).

O desvio padrão e a média tiveram praticamente valores próximos para os movimentos de ECD e ECE. Esses valores podem ser devido aos atletas A (0.5), B (0.5) e D (1.0) terem uma lesão cervical alta e não terem a musculatura do tríceps preservado.

Os pontos baixos (0.5 e 1.0) apresentaram espessura muscular semelhante a dos pontos altos $(2.0,2.5,3.0)$ nos movimentos de FCD e FCE, onde A (0.5) apresentou a mesma espessura muscular que I (2.5) no movimento de FCD e B (0.5) apresentou maior espessura muscular que $H(2.5)$, também no movimento de FCD. Essa semelhança ou superioridade da espessura muscular dos flexores do cotovelo dos pontos baixos podem ser explicadas pela musculatura ser preservada para ambos os atletas e, além disso, a biomecânica do movimento utilizada para a propulsão da cadeira de rodas no ponto baixo proporciona maior trabalho dos flexores do cotovelo e para os pontos altos os extensores do cotovelo são mais utilizados.

$\mathrm{O}$ atleta $\mathrm{D}(1.0)$ apresentou $\mathrm{ON}$ de força muscular no teste de força isométrica máxima para ECD e ECE, porém apresentou a maior espessura muscular no $\operatorname{ECD}(3,02 \mathrm{~cm})$ de toda a amostra. A hipótese é que pode haver tecido adiposo intramuscular que não pode ser mensurado pelo equipamento US. 
A correlação bi-serial (point bi-serial coefficient) utilizada para analisar os níveis de força muscular isométrica máxima entre os jogadores de ataque (2.0, 2.5 e 3.0) e defesa (0.5 e 1.0), apresentou significância estatística para os movimentos de ECD ( $p \leq 0,009)$ e para ECE $(p \leq 0,01)$, lembrando que a significância estatística foi determinada para $p \leq 0,05$. Resultados esses que podem ser explicados novamente pelo déficit do tríceps nos pontos baixos. Já ao utilizar a correlação de Spearman para analisar a força isométrica máxima do membro dominante e não dominante, vemos que para todos os movimentos (flexão e extensão de ombro e flexão e extensão de cotovelo) apresentaram significância estatística onde é $p \leq 0,05$.

A correlação entre a musculatura agonista e antagonista também foi analisada pelo teste de Spearman. Dessa maneira, a significância estatística foi encontrada tanto para os movimentos de FOD x FOE $(p \leq 0.001)$ e EOD x EOE $(p \leq 0.008)$. Assim sendo, a hipótese de não ter tido correlação estatisticamente significativa para os movimentos de cotovelo fica novamente sendo a falta de funcionalidade do tríceps.

Para análise de força muscular isométrica máxima e espessura muscular entre os jogadores titulares e reservas foi utilizada a comparação bivariada (Test Mann Whitney U). Onde os jogadores titulares (C (1.0), E (2.0), H (2.5) e I (2.5) somando um total de 8 pontos permitidos pelo regulamento da IWRF (2011) foram os principais jogadores que participaram do último campeonato disputado pela ADEACAMP no ano de 2015 e os jogadores reservas foram os jogadores que complementaram a equipe ao longo do ano de 2015. Assim sendo, dos 8 movimentos utilizados para o teste de força (FOD, FOE, EOD, EOE, FCD, FCE, ECD e ECE) 6 apresentaram significância estatística (FOD, FOE, EOD, EOE, FCE e ECE). Já para a espessura muscular somente o FCE apresentou significância estatística, sendo que os maiores valores foram referentes aos jogadores reservas. Isso mostra que a força pode ser uma variável que está diretamente ligada ao desempenho esportivo de atletas de RCR, já a espessura muscular pode mascarar o resultado de força devido aos tecidos adiposos intramuscular.

\section{CONCLUSÃO}

Com o presente estudo, pode-se concluir que quanto maior a classificação funcional do atleta, maiores são os valores de força muscular isométrica máxima. Além de permitir que fosse observado que a força isométrica mostrou ter correlação com o desempenho dos atletas de RCR.

\section{REFERÊNCIAS}

1. International Wheelchair Rugby Federation. Manual de classificação da IWRF [texto na Internet]. Sheffield: IWRF; c2011 [citado 2016 Ago 11]. Disponível em: http://www.iwrf.com/resources/iwrf docs/IWRF Classificat ion Manual 3rd Edition rev-2011 \%28Portuguese\%29.pdf

2. International Wheelchair Rugby Federation. International rules for the sport of wheelchair rugby (Portuguese) [Text on the Internet]. Richmond: IWRF; c2015 [cited 2016 Aug 08]. Available from: http://www.iwrf.com/resources/iwrf docs/Wheelchair Rug by International Rules 2015 \%28Portuguese\%29.pdf
3. Machado ABM. Neuroanatomia funcional. Rio de Janeiro: Atheneu; 1986.

4. Taricco MA. Etiologia das lesões medulares. In: Greve JMD, Casallis MEP, Barros Filho TEP. Diagnostico e tratamento da lesão medula da espinhal. São Paulo: Roca; 2001. p. 1-8.

5. Valent L, Dallmeijer A, Houdijk H, Talsma E, van der Woude L. The effects of upper body exercise on the physical capacity of people with a spinal cord injury: a systematic review. Clin Rehabil. 2007;21(4):315-30. Doi: https://doi.org/10.1177/0269215507073385

6. Ribeiro Neto F, Guanais P, Lopes GH, Dornelas E, Campos Barbetta D, Coutinho AC, et al. Influence of Relative Strength on Functional Independence of Patients With Spinal Cord Injury. Arch Phys Med Rehabil. 2017;98(6):1104-12.

Doi:

https://doi.org/10.1016/j.apmr.2016.08.483

7. Nash MS. Exercise as a health-promoting activity following spinal cord injury. J Neurol Phys Ther. 2005;29(2):87-103.

https://doi.org/10.1097/01.npt.0000282514.94093.c6

8. Pelletier CA, Hicks AL. Muscle fatigue characteristics in paralyzed muscle after spinal cord injury. Spinal Cord. 2011;49(1):125-30.

Doi: https://doi.org/10.1038/sc.2010.62

9. Biering-Sørensen B, Kristensen IB, Kjaer M, BieringSørensen F. Muscle after spinal cord injury. Muscle Nerve. 2009;40(4):499-519.

Doi: https://doi.org/10.1002/mus.21391

10. Thomas JR, Nelson JK, Silverman SJ. Métodos de pesquisa em atividade física. 6 ed. Porto Alegre: Artmed; 2012.

11. Bohannon RW. Reference values for extremity muscle strength obtained by hand-held dynamometry from adults aged 20 to 79 years. Arch Phys Med Rehabil. 1997;78(1):26-32. Doi: https://doi.org/10.1016/s00039993(97)90005-8

12. Reeves Reeves ND, Maganaris CN, Narici MV. Ultrasonographic assessment of human skeletal muscle size. Eur J Appl Physiol. 2004;91(1):116-8. Doi: https://doi.org/10.1007/s00421-003-0961-9

13. Abe T, DeHoyos DV, Pollock ML, Garzarella L. Time course for strength and muscle thickness changes following upper and lower body resistance training in men and women. Eur J Appl Physiol. 2000;81(3):174-80. Doi: https://doi.org/10.1007/s004210050027

14. Ogasawara R, Thiebaud RS, Loenneke JP, Loftin M, Abe T. Time course for arm and chest muscle thickness changes following bench press training. Interv Med Appl Sci. 2012;4(4):217-20.

Doi:

https://doi.org/10.1556/IMAS.4.2012.4.7

15. Campana MB. O rugbi em cadeira de rodas = aspectos tecnicos e taticos e diretrizes para seu desenvolvimento [Dissertação]. Campinas: Universidade Estadual de Campinas; 2010.

16. Campos LFCC, Pena LGS, Gouveia RB, Gatti AMM, Paranhos VMS, Gorla Jl. Rugby em cadeira de rodas: aspectos relacionados a caracterização, controle e avaliação. Conexões. 2013;11(4):72-89. Doi: https://doi.org/10.20396/conex.v11i4.8637591 
17. Gouveia RB. Avaliação do desempenho anaeróbio de atletas de Rugby em cadeira de rodas [Dissertação]. Campinas: Universidade Estadual de Campinas; 2013.

18. Morgulec-Adamowicz N, Kosmol A, Molik B, Yilla AB, Laskin JJ. Aerobic, anaerobic, and skill performance with regard to classification in wheelchair rugby athletes. Res Q Exerc Sport. 2011;82(1):61-9. Doi: https://doi.org/10.1080/02701367.2011.10599722

19. Goosey-Tolfrey V, Castle P, Webborn N, Abel T. Aerobic capacity and peak power output of elite quadriplegic games players. Br J Sports Med. 2006;40(8):684-7. Doi: https://doi.org/10.1136/bjsm.2006.026815

20. Sporner ML, Grindle GG, Kelleher A, Teodorski EE, Cooper $\mathrm{R}$, Cooper RA. Quantification of activity during wheelchair basketball and rugby at the National Veterans Wheelchair Games: A pilot study. Prosthet Orthot Int. 2009;33(3):2107. Doi: https://doi.org/10.1080/03093640903051816

21. Yilla $A B$, Sherril C. Validation the Beck Battery of quad Rugby skill tests. Adapt Phys Activ Q. 1998;15(2):155-67. Doi: https://doi.org/10.1123/apaq.15.2.155

22. Poulain $M$, Vinet $A$, Bernard PL, Varray A. Reproducibility of the Adapted Leger and Boucher Test for wheelchairdependent athletes. Spinal Cord. 1999;37(2):129-35. Doi: https://doi.org/10.1038/sj.sc.3100774
23. Gorla JI, Costa e Silva AA, Costa LT, Campos LFCC. Validação da bateria "Beck" de testes de habilidades para atletas brasileiros de "rugby" em cadeira de rodas. Rev Bras Educ Fís Esporte. 2011;25(3):473-86. Doi: http://dx.doi.org/10.1590/S1807-55092011000300011

24. Hartung GH, Lally DA, Blancq RJ. Comparison of treadmill exercise testing protocols for wheelchair users. Eur J Appl Physiol Occup Physiol. 1993;66(4):362-5. Doi: http://dx.doi.org/10.1007/BF00237783

25. Burns SP, Breuninger A, Kaplan C, Marin H. Hand-held dynamometry in persons with tetraplegia: comparison of make- versus break-testing techniques. Am J Phys Med Rehabil. 2005;84(1):22-9. Doi: https://doi.org/10.1097/01.phm.0000150790.99514.c6

26. Ishida Y, Carroll JF, Pollock ML, Graves JE, Leggett SH. Reliability of B-mode ultrasound for the measurement of body fat and muscle thickness. Am J Hum Biol. 1992;4(4):511-520. Doi: https://doi.org/10.1002/ajhb.1310040410

27. Hislop HJ, Montgomery J. Daniels and worthingham's muscle testing: techniques of manual examination. $6^{\text {th }}$ ed. Philadelphia (PA): WB Saunders; 2002. 